\title{
QUASI-EXPECTATIONS AND AMENABLE VON NEUMANN ALGEBRAS
}

\author{
JOHN W. BUNCE AND WILLIAM L. PASCHKE ${ }^{1}$
}

\begin{abstract}
A quasi-expectation of a $C^{*}$-algebra $A$ on a $C^{*}$-subalgebra $B$ is a bounded linear projection $Q: A \rightarrow B$ such that $Q(x a y)=x Q(a) y \forall x, y \in$ $B, a \in A$. It is shown that if $M$ is a von Neumann algebra of operators on Hilbert space $H$ for which there exists a quasi-expectation of $B(H)$ on $M$, then there exists a projection of norm one of $B(H)$ on $M$, i.e. $M$ is injective. Further, if $M$ is an amenable von Neumann subalgebra of a von Neumann algebra $N$, then there exists a quasi-expectation of $N$ on $M^{\prime} \cap N$. These two facts yield as an immediate corollary the recent result of $A$. Connes that all amenable von Neumann algebras are injective.
\end{abstract}

Let $A$ be a unital $C^{*}$-algebra and $B$ a unital $C^{*}$-subalgebra of $A$. By a quasi-expectation of $A$ on $B$, we mean a bounded linear projection $Q: A \rightarrow B$ such that $Q(x a y)=x Q(a) y \forall x, y \in B, a \in A$. A classical result of $\mathrm{J}$. Tomiyama [13] says that any projection of norm one of $A$ onto $B$ is a quasi-expectation. In what follows, we will show that if $M$ is a von Neumann algebra of operators on Hilbert space $H$, then the existence of a quasi-expectation of $B(H)$ on $M$ implies the existence of a projection of norm one of $B(H)$ on $M$. We will show further that if $M$ is an amenable von Neumann subalgebra (as in [2]) of a von Neumann algebra $N$, then there exists a quasi-expectation of $N$ on $M^{\prime} \cap N$. These two results yield as an immediate corollary the recent theorem of A. Connes [3] stating that amenable von Neumann algebras are injective.

We begin with an elementary result relating the existence of projections of norm one on a finite, countably decomposable von Neumann algebra $M$ to $M$-centralizing extensions of traces on $M$. For a functional $f$ on a ${ }^{*}$-algebra $B$ and $x \in B$, we write $x \cdot f, f \cdot x$, and $f^{*}$ for the functionals on $B$ defined respectively by $(x \cdot f)(y)=f(y x),(f \cdot x)(y)=f(x y)$, and $f^{*}(y)=\overline{f\left(y^{*}\right)}$. A trace on $B$ is a nonzero positive functional $\tau$ such that $\tau \cdot x=x \cdot \tau \forall x \in B$.

Proposition 1. Let $A$ be a unital $C^{*}$-algebra and $M$ a finite, countably decomposable von Neumann algebra contained unitally in $A$. The following are equivalent:

Received by the editors June 1, 1977.

AMS (MOS) subject classifications (1970). Primary 46L05, 46L10.

Key words and phrases. Amenable, injective, normal and singular parts, projection of norm one, quasi-expectation.

${ }^{1}$ Both authors partially supported by a grant from the National Science Foundation. 
(i) There is a functional $f \in A^{*}$ satisfying $x \cdot f=f \cdot x \forall x \in M$ such that $\left.f\right|_{M}$ is a faithful positive functional on $M$.

(ii) There is a positive functional $g$ on A satisfying $g \cdot x=x \cdot g \forall x \in M$ such that $\left.g\right|_{M}$ is faithful.

(iii) There is a projection $E$ of norm one of $A$ on $M$.

Proof. We first recall some facts from [10] and [11] concerning the decomposition of a functional in $M^{*}$ into normal and singular parts. Regard $M_{*}$, the predual of $M$, as a subspace of $M^{*}$. The annihilator of $M_{*}$ in the von Neumann algebra $M^{* *}$ has the form $M^{* *}\left(1-z_{0}\right)$ for some central projection $z_{0}$ of $M^{* *}$. Let $j: M \rightarrow M^{* *}$ be the natural injection. For $f \in M^{*}$, define $f_{n}$, $f_{s} \in M^{*}$ by $f_{n}(x)=\left\langle f, j(x) z_{0}\right\rangle$ and $f_{s}(x)=\left\langle f, j(x)\left(1-z_{0}\right)\right\rangle$. We have $f=f_{n}$ $+f_{s}$, with $f_{n}, f_{s} \geqslant 0$ if $f \geqslant 0$, and $(f \cdot x)_{n}=f_{n} \cdot x,(x \cdot f)_{n}=x \cdot f_{n} \forall x \in M$. Moreover, $\left(M^{*}\right)_{n}=M_{*}$ and the functionals $g$ in the complementary subspace $M_{s}^{*}$ are characterized by the property that every nonzero projection in $M$ majorizes a nonzero projection in the kernel of $g$.

To show that (i) implies (ii), we argue as in [3]. We may assume that $f=f^{*}$, since $f+f^{*}$ satisfies (i) also. Let $f=f^{+}-f^{-}$be the Jordan decomposition of $f$, with $f^{+}, f^{-} \geqslant 0$ and $\left\|f^{+}\right\|+\left\|f^{-}\right\|=\|f\|$. For any unitary $u \in M$, we have $u^{*} \cdot f \cdot u=f$, so by the uniqueness of the Jordan decomposition we must have $u^{*} \cdot f^{+} \cdot u=f^{+}$. Since $\left.f^{+}\right|_{M} \geqslant\left. f\right|_{M}$ and the latter is positive and faithful by assumption, we may take $g=f^{+}$.

To show that (ii) implies (iii), we define $\theta: A \rightarrow M_{*}$ by $\theta(a)=\left(\left.(g \cdot a)\right|_{M}\right)_{n}$. If $a \in A^{+}$, then $\left.(g \cdot a)\right|_{M} \geqslant 0$ because for any $x \in M$ we have $(g \cdot a)\left(x^{*} x\right)$ $=g\left(a x^{*} x\right)=g(|x| a|x|) \geqslant 0$. Hence $\theta(a) \geqslant 0 \forall a \in A^{+}$. Let $\tau_{0}=\theta(1)$. We claim that $\tau_{0}$ is faithful. Indeed, let $\phi=\left.g\right|_{M}$, so $\phi=\tau_{0}+\phi_{s}$. For any nonzero projection $p$ there is a nonzero $q \leqslant p$ with $\phi_{s}(q)=0$. We are assuming that $\phi$ is faithful, so $\tau_{0}(p) \geqslant \tau_{0}(q)=\phi(q)>0$; this shows that $\tau_{0}$ is faithful. Further, we have for any $x \in M$ that $\tau_{0} \cdot x=\left(\left.g\right|_{M}\right)_{n} \cdot x=\left(\left.g\right|_{M} \cdot x\right)_{n}=\left(\left.(g \cdot x)\right|_{M}\right)_{n}=$ $\left(\left.(x \cdot g)\right|_{M}\right)_{n}=x \cdot \tau_{0}$, so $\tau_{0}$ is a (faithful, normal) trace on $M$. We have shown also that $\theta(x)=\tau_{0} \cdot x \forall x \in M$. If $a \in A^{+}$, then $a \leqslant\|a\| 1$, so $0 \leqslant \theta(a) \leqslant$ $\|a\| \tau_{0}$. By S. Sakai's Radon-Nikodým theorem (1.24.3 of [9]), there exists $h \in M^{+}$such that $\theta(a)=h^{1 / 2} \cdot \tau_{0} \cdot h^{1 / 2}\left(=\tau_{0} \cdot h\right.$ because $\tau_{0}$ is a trace). We conclude that for any $b \in A$, there exists $E(b) \in M$ such that $\theta(b)=\tau_{0}$. $E(b)$. This $E(b)$ is unique because $\tau_{0}$ is faithful. We have $E(x)=x \forall x \in M$, so $E$ is a projection of $A$ onto $M$. Since $E(1)=1$ and $E$ is positive, the norm of $E$ is 1 .

That (iii) implies (i) follows from the fact that $E$ is a quasi-expectation. To obtain $f$, let $\tau$ be a faithful normal trace on $M$ and let $f=\tau \circ E$.

We remark that the most interesting special case of Proposition 1 (namely when $M$ is a von Neumann algebra of operators on Hilbert space $H$ and $A=B(H)$ ) has been obtained by A. Connes [3] using more sophisticated methods; the argument appears at the end of the proof of the semifinite case of Theorem 1 of [3], and consists of showing that $M$ is semidiscrete in the sense of [5]. Also, G. Robertson has recently shown that (ii) implies (iii) if the 
restriction of the positive functional $g$ in (ii) to $M$ is assumed to be normal. (See the proof of 1.2 in [8].)

A von Neumann algebra $M$ of operators on Hilbert space $H$ is said to be injective [2] if there exists a projection of norm one of $B(H)$ on $M$. Injectivity is an algebraic invariant of $M$ and is equivalent to the existence of a projection of norm one on $M^{\prime}$. (Use Tomita's theorem to generalize 4.2 of [6].)

THEOREM 2. Let $M$ be a von Neumann algebra of operators on Hilbert space $H$. If there exists a quasi-expectation of $B(H)$ on $M$, then $M$ is injective.

Proof. Let $Q: B(H) \rightarrow M$ be a quasi-expectation. If $M$ is finite and countably decomposable, let $\tau$ be a faithful trace on $M$ and define $f \in$ $B(H)^{*}$ by $f=\tau \circ Q$. This $f$ satisfies (i) of Proposition 1 , so the theorem is proved for finite, countably decomposable $M$.

Now suppose that $M$ is semifinite. There is an increasing net $\left\{e_{\alpha}\right\}$ of projections in $M$ with $e_{\alpha} \uparrow 1$ and $e_{\alpha} M e_{\alpha}$ finite and countably decomposable $\forall \alpha$. (Argue as in III,2,3 of [4] to see that the set of finite, countably decomposable projections of $M$ is directed upward.) Since $Q\left(e_{\alpha} B(H) e_{\alpha}\right)=$ $e_{\alpha} M e_{\alpha}, Q$ defines a quasi-expectation of $e_{\alpha} B(H) e_{\alpha}$ on $e_{\alpha} M e_{\alpha}$ by restriction. For each $\alpha$, then, there is a projection $E_{\alpha}$ of norm one of $e_{\alpha} B(H) e_{\alpha}$ on $e_{\alpha} M e_{\alpha}$. Denote by $D$ the directed set which indexes $\left\{e_{\alpha}\right\}$. Let $L$ be a state of the $C^{*}$-algebra $l^{\infty}(D)$ such that $L\left(\left\{c_{\alpha}\right\}\right)=c$ whenever $\left\{c_{\alpha}\right\}$ is a net in $\mathbf{C}$ indexed by $D$ such that $\lim _{\alpha} c_{\alpha}=c$. For $T \in B(H)$, define $E(T) \in B(H)$ by $(E(T) \xi, \eta)=L\left(\left\{\left(E_{\alpha}\left(e_{\alpha} T e_{\alpha}\right) \xi, \eta\right)\right\}\right)(\xi, \eta \in H)$. It is easily checked that $E$ is a projection of norm one of $B(H)$ on $M$, so we are finished with the semifinite case.

Quasi-expectations respect direct sum decompositions, and the direct sum of two von Neumann algebras is injective if and only if each summand is, so to conclude the proof of the theorem we may assume that $M$ is properly infinite. By 8.2 and 4.6 of [12], $M$ is the crossed product $W^{*}(N, \mathbf{R}, \alpha)$ of a semifinite von Neumann algebra $N$ by an ultraweakly continuous action $\alpha$ of $\mathbf{R}$ on $N$. Consider the dual action $\hat{\alpha}$ of $\hat{\mathbf{R}}=\mathbf{R}$ on $M$ ( $\$ 4$ of [12]); the fixed-point algebra $M_{\hat{\alpha}}$ of $M$ under $\hat{\alpha}$ is precisely $N$ by 7.1 of [12]. Let $\mu$ be an invariant mean on $l^{\infty}(R)$. One checks without difficulty that $(F(x) \xi, \eta)=$ $\mu_{(t)}\left\{\left(\hat{\alpha}_{t}(x) \xi, \eta\right)\right\}(x \in M, \xi, \eta \in H)$ defines a projection $F$ of norm one of $M$ onto $M_{\hat{\alpha}}=N$. Since $F \circ Q$ is a quasi-expectation of $B(H)$ on $N$, it follows from the previous paragraph that $N$ is injective. Now $M$ is generated by $N$ and a one-parameter group $\left\{U_{t}: t \in \mathbf{R}\right\}$ of unitaries normalizing $N$, so $M^{\prime}=N^{\prime} \cap U_{\mathbf{R}}^{\prime}$. If we let $G$ be a projection of norm one of $B(H)$ on $N^{\prime}$ and take $\mu$ as above, $(E(T) \xi, \eta)=\mu_{(t)}\left\{\left(U_{t} G(T) U_{-t} \xi, \eta\right)\right\}(T \in B(H), \xi, \eta \in H)$ defines a projection of norm one of $B(H)$ on $M^{\prime}$, so $M$ is injective.

Let $M$ be a von Neumann algebra. A dual normal Banach $M$-bimodule [7] is a Banach $M$-bimodule $X$ such that $X$ is the conjugate space of a Banach space $X_{*}$, and for each $\phi \in X_{*}$, the maps $(b, x) \rightarrow \phi(b \cdot x), \phi(x \cdot b)$ from 
$M \times X$ to $C$ are ultraweakly continuous in $b$ (for fixed $x \in X$ ) and $\sigma\left(X, X_{*}\right)$-continuous in $x$ (for fixed $\left.b \in M\right)$. As in [2], we call $M$ amenable (as a von Neumann algebra) if every bounded derivation $\delta$ of $M$ in a dual normal Banach $M$-bimodule $X$ has the form $\delta(b)=x \cdot b-b \cdot x$ for some $x \in X$.

THEOREM 3. Let $N$ be a von Neumann algebra and $M$ a von Neumann subalgebra of $N$. If $M$ is amenable, then there is a quasi-expectation of $N$ on $M^{\prime} \cap N$.

Proof. Let $Y_{*}=N \hat{\otimes} N_{*}$, the completion in the greatest cross norm of the algebraic tensor product of $N$ with its predual, and let $Y=\left(Y_{*}\right)^{*}$. We may identify $Y$ with $B(N)$, the bounded linear endomorphisms of $N$, by the pairing

$$
\langle G, T \otimes \psi\rangle=\psi(G(T)) \quad\left(G \in B(N), T \in N, \psi \in N_{*}\right) .
$$

(Henceforth, we will use the letters $G, T, \psi, b$, and $s$ to denote generic elements of $Y, N, N_{*}, M$, and $M^{\prime} \cap N$, respectively.) Define left and right actions of $M$ on $Y$ by $(b \cdot G)(T)=b G(T),(G \cdot b)(T)=G(T) b$. It is clear that these actions make $Y$ into a Banach $M$-bimodule. Since $\langle G \cdot b, T \otimes \psi\rangle$ $=\psi(G(T) b)=\langle G, T \otimes b \cdot \psi\rangle$ and $\langle b \cdot G, T \otimes \psi\rangle=\psi(b G(T))=\langle G, T \otimes$ $\psi \cdot b\rangle$, it is clear that the module actions on both sides satisfy the continuity conditions required for $Y$ to be a dual normal Banach $M$-bimodule. Let $X=\{G \in Y:\langle G, T s \otimes \psi-T \otimes s \cdot \psi\rangle=0 ;\langle G, s T \otimes \psi-T \otimes \psi \cdot s\rangle=$ $\left.0 ;\langle G, s \otimes \psi\rangle=0 \forall s \in M^{\prime} \cap N, T \in N, \psi \in N_{*}\right\}$. It is readily checked that $X$ is a submodule of $Y$; since $X$ is $\sigma\left(Y, Y_{*}\right)$-closed, $X$ is a dual normal Banach $M$-bimodule. Let $J: N \rightarrow N$ be the identity map, and define $\delta$ : $M \rightarrow Y$ by $\delta(b)=J \cdot b-b \cdot J$. We have $\langle J, T \otimes \psi\rangle=\psi(T)$, whence it follows that $J \cdot b$ and $b \cdot J$ annihilate all $\Phi \in Y_{*}$ of the form $T s \otimes \psi-T \otimes s$ $\cdot \psi$ or $s T \otimes \psi-T \otimes \psi \cdot s$. Also, $\langle\delta(b), s \otimes \psi\rangle=\langle J, s \otimes b \cdot \psi\rangle-\langle J, s \otimes \psi$ $\cdot b\rangle=\psi(s b)-\psi(b s)=0$. We conclude that $\delta(M) \subseteq X$, i.e. $\delta$ is a bounded derivation of $M$ in $X$. Since $M$ is amenable, there exists $H \in X$ such that $\delta(b)=H \cdot b-b \cdot H$. Let $Q=J-H$. We claim that $Q$ is a quasiexpectation of $N$ on $M^{\prime} \cap N$. Because $H \in X$, we have $H\left(M^{\prime} \cap N\right)=0$ by the third criterion for membership in $X$, so $Q$ fixes $M^{\prime} \cap N$. Further, $\psi(H(T) s)=\langle H, T \otimes s \cdot \psi\rangle=\langle H, T s \otimes \psi\rangle=\psi(H(T s))$ by the first criterion, so $H(T) s=H(T s)$. Similarly, the second criterion insures that $H(s T)=s H(T)$. Thus $Q\left(s_{1} T s_{2}\right)=s_{1} Q(T) s_{2} \forall T \in N, s_{1}, s_{2} \in M^{\prime} \cap N$. Finally, $Q \cdot b-b \cdot Q=\delta(b)-\delta(b)=0$, since $J$ and $H$ both implement $\delta$, i.e. $Q(N) \subseteq M^{\prime} \cap N$, and we conclude that $Q$ is a quasi-expectation of $N$ on $M^{\prime} \cap N$.

Letting $N=B(H)$ in Theorem 3 and applying Theorem 2, we have

Corollary 4 (Connes [3]). Amenable von Neumann algebras are injective.

If $A$ is a $C^{*}$-algebra such that $A^{* *}$ is injective, then $A$ is nuclear [1]. The following corollary then follows easily (as in the proof of Corollary 2 of [3]). 
Corollary 5 (CONNES [3]). Amenable $C^{*}$-algebras are nuclear.

\section{REFERENCES}

1. M. D. Choi and E. G. Effros, Nuclear $C^{*}$-algebras and injectivity: the general case, Indiana Univ. Math. J. 26 (1977), 443-446.

2. A. Connes, Classification of injective factors, Ann. of Math. 104 (1976), 73-115.

3. On the cohomology of operator algebras, preprint, 1976.

4. J. Dixmier, Les algèbres d'opérateurs dans l'espace hilbertien, 2nd ed., Gauthier-Villars, Paris, 1969.

5. E. G. Effros and E. C. Lance, Tensor products of operator algebras, Advances in Math. 25 (1977), 1-34.

6. J. Hakeda and J. Tomiyama, On some extension properties of von Neumann algebras, Tôhoku Math. J. 19 (1967), 315-323.

7. R. V. Kadison and J. Ringrose, Cohomology of operator algebras. I, Acta Math. 126 (1971), 227-243.

8. G. Robertson, States which have a trace-like property relative to a $C^{*}$-subalgebra of $B(H)$, Glasgow Math. J. 17 (1976), 158-160.

9. S. Sakai, $C^{*}$-algebras and $W^{*}$-algebras, Springer-Verlag, Berlin and New York, 1971.

10. M. Takesaki, On the conjugate space of operator algebra, Tôhoku Math. J. 10 (1958), $194-203$.

11. Acad. 35 (1959), 365-366.

12. __ Duality for crossed products and the structure of von Neumann algebras of type III, Acta Math. 131 (1973), 249-310.

13. J. Tomiyama, On the projection of norm one in $W^{*}$-algebras, Proc. Japan Acad. 33 (1957), 608-612.

Department of Mathematics, University of Kansas, LaWrence, Kansas 66045 